\title{
INVERSE STURM-LIOUVILLE PROBLEM FOR A STAR GRAPH BY THREE SPECTRA
}

\author{
VYACHESLAV PIVOVARCHIK
}

Abstract. A three spectra problem for a star graph of three edges is solved. The given data are 1) the spectrum of a boundary value problem on the whole graph with the Dirichlet boundary conditions at the pendant vertices, continuity and Kirchhoff's conditions at the interior vertex, 2) the spectrum of the Dirichlet-Neumann problem on one of the edges, 3) the spectrum of the Dirichlet-Dirichlet problem on the union of two other edges. The aim is to find the potentials on the edges. Conditions on three sequences of numbers are found sufficient to be the spectra of these three problems.

Mathematics subject classification (2010): 34B45, 34A55.

Keywords and phrases: eigenvalue, spectral problem, Dirichlet boundary condition, Neumann boundary condition, Marchenko equation, Lagrange interpolation series, sine-type function, essentially positive Nevanlinna function.

\section{REFERENCES}

[1] S. AleXANDer Superconductivity of networks. A percolation approach to the effects of disorder, Phys. Rev. B 27, (1983), 1541-1557.

[2] J. VON BELOW, Can one here the shape of a network?, Partial differential equations on multistructures (Luminy, 1999), Lecture Notes in Pure and Appl. Math. 219, Dekker, New York, 2001, 19-36.

[3] G. Berkolaiko and P. Kuchment Introduction to Quantum Graphs, Math. Surveys Monograps, AMS 186, Providence, 2012

[4] O. BOYKo, O. MARTYNYUK AND V. PIVOVARChIK, On connection between multiplicities of eigenvalues in finitedimensional and infinitedimensional problems on graphs, Ukrainian Mathematical Journal 69, 4 (2017), 445-455.

[5] M. Brown and R. Weikard, A Borg-Levinson theorem for trees, Proc. R. Soc. Lond. Ser. A Math. Phys. Eng. Sci. 461, 2062 (2005) 3231-3243.

[6] C. Cattaneo, The spectrum of the continuous Laplacian on a graph, Monatsh. Math. 23 (1997), $1-30$.

[7] R. CARlson, Adjoint and self-adjoint differential operators on graphs, Electron. J. Diff. Eqns. 6 (1998) $10 \mathrm{pp}$. (electronic).

[8] R. Carlson And V. Pivovarchik, Ambarzumian's theorem for trees, Electron. J. Diff. Eqns., 2007, (2007), 142, 1-9.

[9] R. Courant And D. Hilbert, Methods of Mathematical Physics, 1 Interscience Publishers, Inc., N.Y., 1953.

[10] S. CURRIE AND B. WATSON Eigenvalue asymptotics for differential operators on graphs, J. Comp., Appl. Math., 182 (2005) 13-31.

[11] P. EXNER, Weakly coupled states on branching graphs, Lett. Math. Phys. 38 (1996), 313-320.

[12] P. EXNER, A duality between Schrödinger operators on graphs and certain Jacobi matrices, Ann.Inst. H. Poincaré, Sec. A, 66, 4 (1997), 359-371.

[13] J. Genin And J. S. Maybee, Mechanical vibration trees, J. Math. Anal. Appl. 45 (1974), 746-763.

[14] G. Gladwell, Inverse problems in vibration, Kluwer Academic Publishers, Dordrecht, 2004.

[15] G. Gladwell, Matrix inverse eigenvalue problems, In: G. Gladwell, A. Morassi, eds., Dynamical Inverse Problems: Theory and Applications, CISM Courses and Lectures 529 (2011) 1-29. 
[16] B. Gutkin And U. Smilansky, Can one hear the shape of a graph?, J. Phys. A: Math. Gen. 34 (2001), 6061-6068.

[17] I. KaC AND V. PivovarchiK, On multiplicity of quantum graph spectrum, J. Phys. A: Math. Theor. 44 (2011), 105301.

[18] M. KISs, An Ambarzumian type theorem on graphs, arXiv:1610.00971v2 [math.SP].

[19] V. Kostrikin And R. Schrader, Kirchhoff's rule for quantum wires, J. Phys. A: Math. Gen. 32, 1 (1999), 595-630

[20] P. KuChment, Quantum graphs: an introduction and a brief survey, in: 'Analysis on Graphs and its Applications', Proc. Symp. Pure Math. AMS (2008), 291-314.

[21] P. KURASOV, Schrödinger operators on graphs and geometry I: Essentially bounded potentials, J. Func. Anal 254 (2008), 934-953.

[22] Y. LATUSHKIn AND V. PIVOVARCHIK, Scattering in a Forked-Shaped Waveguide. Integral Equations and Operator Theory, 61 3, (2008), 365-399.

[23] B. YA. LEVIN AND YU. I. LYUBARSKII, Interpolation by entire functions belonging to special classes and related expansions in series of exponentials, (in Russian), Izv. Akad. Nauk SSSR Ser. Mat., 39, (1975), 3, 657-702, 704, English translation Math. USSR-Izv., 9, (1975), 3, 621-662.

[24] B. M. LeVitan And M. G. GaS YMOV, Determination of a differential equation by two of its spectra, (in Russian), Uspekhi Mat. Nauk, 19, 2 (116) (1964), 3-63.

[25] J. A. LuBARY, On the geometric and algebraic multiplicities for eigenvalue problems on graphs, in Lecture Notes in Pure and Appl. Math. (Marcel Dekker, New York, I (2001), 219, 135-146.

[26] V. A. MARCHENKo, Introduction to the theory of inverse problems of spectral analysis, (in Russian), Acta, Kharkov, 2005.

[27] V. A. Marchenko, Sturm-Liouville operators and applications, (in Russian), Naukova Dumka, Kiev, 1977, English translation: Oper. Theory Adv. Appl., 22, Birkhäuser Verlag, Basel, 1986.

[28] M. Möller ANd V. Pivovarchik, Spectral Theory of Operator Pencils, Hermite-Biehler Functions, and their Applications, Operator Theory: Advances and Applications, 264, Birkhäuser, Basel, 2015.

[29] V. PIVOVARCHIK, Inverse problem for the Sturm-Liouville equation on a simple graph, SIAM J. Math. Anal. 32 (2000), 801-819.

[30] V. PIVOVARCHIK, Inverse problem for the Sturm-Liouville equation on a star-shaped graph, Math. Nachr. 280, 13-14 (2007), 1595-1619.

[31] W. Rundell AND P. S ACKS, Inverse Eigenvalue Problem for a simple stargraph, J. Spectral Theory 5, (2015), 2, 363-380.

[32] S. Seshu And M. B. Reed, Linear Graphs and Electrical Circuits, Addison-Wesley Publishing Co., Inc., London, 1961.

[33] C.-F. YANG AND X.-C. XU, Ambarzumyan-type theorems on graphs with loops and double edges, J. Math. Ana., Appl., 444, (2016), 15, 1348-1358.

[34] R. M. Young, An Introduction to Nonharmonic Fourier Series, Acad. Press, New York, 1980.

[35] V. YURKO, Inverse spectral problems for Sturm-Liouville operators on graphs, Inverse Problems 21 (2005), 1075-1086. 